Anderer gegen ihn, ganz für seine Wissenschaft, sein Lehramt, seine Familie und seine Freunde.

Buchner hat kein besonders hohes Alter erreicht. Seine rastlose Thätigkeit, der beständige Drang zur Arbeit schwächten seine nicht sehr kräftige Gesundheit; am 6. Juni 1852 unterlag er einem chronischen Leiden im 70. Lebensjahre.

Buchners Verdienste um die Pharmacie können in wenige Worte zusammengefasst werden. Er hat, wie Pettenk ofer an dessen Grabe so trefflich aussprach, die Idee verfolgt, das Apothekergewerbe durch strenge Wissenschaftlichkeit in seinen Grundlagen zu adeln. Die geistige Hebung des Apothekerstandes lag ihm am Herzen; der Verwirklichung dieser Idee hat er seine Gesundheit, sein Leben geopfert. Und wenn einmal die Geschichte der Pharmacie in der ersten Hälfte dieses Jahrhunderts geschrieben wird, so wird darin der Name Johann Andreas Buchner an erster Stelle genannt werden.

\title{
Ueber die quantitative Bestimmung des Eisens in allen gebräuchlichen Eisenpräparaten auf jodometrischem Wege.
}

\author{
Von Dr. C. Schacht.
}

Ueber ein abgekürztes Verfahren der quantitativen Bestimmung des Eisens im Ferrum carbonicum saccharatum und im Ferrum oxydatum saccharatum solubile auf jodometrischem Wege habe ich in unserer Section auf der 59. Naturforscher-Versammluug eine kurze Mittheilung gemacht, nachdem fast gleichzeitig E. Mylius in Leipzig (Ph. C. 1886, 290/1) und W. Stromeyer jun. in Hannover (Archiv 1886, 542/6) ihre Arbeiten über die Eisenbestimmungen im Ferrum carbonicum saccharatum und im Ferrum oxydatum saccharatum solubile und über die maassanalytische Bestimmung des Eisens in denselben Verbindungen veröffentlicht hatten. Während Mylius eine Aschenbestimmung behufs Feststellung des Eisengehaltes für genügend hält, fällt Stromeyer das Eisensaccharat durch Kochsalz aus und bestimmt das Eisen nach seiner Ueberführung in Eisenchlorid auf jodometrischem Wege. Durch Veraschung kann weder der Eisengehalt des Ferrum carb. sacch. noch das Ferrum oxydat. sacch. solubile bestimmt werden. Man erhält stets zu hohe Werthe, indem 
dem restirenden Oxyd Eisenwerthe entsprechen, welche auf jodometrischem Wege nicht erreicht werden. Ich erhielt bei der Eisenbestimmung des Eisenzuckers nach Mylius 4,45 Proc. Eisenoxyd oder 3,11 Proc. Eisen. Ersteres jodometrisch bestimmt, gab nur 2,80 Proc. Eisen. Beim Ferrum carbonic. saccharat. erhielt ich nach Mylius 9,27 Proc. Eisen, auf jodometrischem Wege nur 9,07 Proc. Das Verfahren von Stromeyer jun. erfordert diverse Manipulationen, wie Abfiltriren des Niederschlages, Auswaschen des Filters und vermeidet auch nicht, wenigstens nicht bei der Eisenbestimmung im Ferrum carbonic. saccharatum, eine der Fehlerquellen, die als Ursache der Nichtübereinstimmung der nach Vorschrift der Ph. Germ. II. erhaltenen Resultate angeführt wird, indem das in dem betreffenden Filtrate enthaltene Eisenchlorür durch Erhitzen mit etwas Kaliumchlorat oxydirt wird und diese Oxydation eine vollständige Verjagung des überschüssigen Chlors nothwendig macht.

Das abgekürzte Verfahren bei der Bestimmung des Eisens im Ferrum oxydatum saccharat. solubile besteht darin, dass dieselbe nach erfolgter Abwägung des Eisensaccharates in demselben Gefässe, am besten in einem mit gutschliessendem Glasstöpsel versehenen Glaskölbchen, zu Ende geführt wird. Auf $1 \mathrm{~g}$ Eisensaccharat bringt man $5 \mathrm{ccm}$ reine Salzsäure von 1,12, lässt das Gemisch $10 \mathrm{Minu-}$ ten lang stehen, fügt $50 \mathrm{ccm}$ Wasser und 0,5 Jodkalium hinzu, lässt eine Stunde in der Wärme stehen und titrirt das ausgeschiedene Jod mit $1 / 10$-Natriumthiosulfatlösung. Es wurden $5 \mathrm{ccm}, 5 \mathrm{ccm}$ und 4,9 ccm verbraucht, also 2,8 Proc., 2,8 Proc. und 2,74 Proc. Eisen gefunden. Handelt es sich um die Eisenbestimmung im Ferrum carbonic. saccharat., so wägt man ca. $0,5 \mathrm{ab}$, bringt dasselbe in ein mit Glasstöpsel versehenes Glaskölbchen, fügt $5 \mathrm{ccm}$ verdünnter Schwefelsäure hinzu und erwärmt dasselbe in einem Wasserbade so lange, bis vollständige Lösung eingetreten ist. Je älter das Präparat ist, um so langsamer löst sich dasselbe. Nach dem Erkalten der klaren Lösung setzt man volumetrische Kaliumpermanganatlösung bis zur vorübergehend bleibenden Röthung hinzu, darauf $1 \mathrm{~g}$ Jodkalium und titrirt nach einstündigem Stehen in der Wärme mit 1/10Natriumthiosulfat das ausgeschiedene Jod. 0,5 gaben $0,0518 \mathrm{~g}$, also 10,3 Proc. Eisen. Von einem anderen Präparate gaben 0,5 g nur $0,0442 \mathrm{~g}$, also 8,84 Proc. Eisen. Nach diesem abgekürzten Verfahren habe ich in folgenden Eisenpräparaten die Eisenbestimmung ausgeführt. 
908 C. Schacht, Quantitative Bestimmung des Eisens auf jodometr. Wege.

Ferrum lacticum.

$1,0500 \mathrm{~g}=0,20272 \mathrm{~g}(36,2 \mathrm{ccm} \times 0,0056)=19,3$ Proc. Fe.

Liquor Ferri acetici.

$3,6690 \mathrm{~g}=0,1624 \mathrm{~g}(29 \mathrm{ccm} \times 0,0056)=4,42$ Proc. Fe.

Man erhielt eine glatte Reaction, wenn man das verdünnte Präparat mit Salzsäure bis zum Sieden erhitzt.

Liquor Ferri oxychlorati.

a. $2,4840 \mathrm{~g}=0,09408 \mathrm{~g}(16,8 \mathrm{ccm} \times 0,0056)=3,78$ Proc. Fe.

b. $3,1450 \mathrm{~g}=0,11704 \mathrm{~g}(20,9 \mathrm{ccm} \times 0,0056)=3,72$ Proc. Fe.

Man verfährt ebenso wie Liquor Ferri acetici.

Liquor Ferri sesquichlorati.

a. $2,5185 \mathrm{~g}=0,25256 \mathrm{~g}(45,1 \mathrm{ccm} \times 0,0056)=10,03$ Proc. Fe.

b. $2,1340 \mathrm{~g}=0,21784 \mathrm{~g}(38,9 \mathrm{ccm} \times 0,0056)=10,20$ Proc. Fe.

Liquor Ferri sulfurici oxydati.

a. $2,0699 \mathrm{~g}=0,21392 \mathrm{~g}(38,2 \mathrm{com} \times 0,0056)=10,33$ Proc. Fe.

b. $2,8275 \mathrm{~g}=0,28728 \mathrm{~g}(51,3 \mathrm{ccm} \times 0,0056)=10,16$ Proc. Fe.

Man erhitzt das Präparat mit Salzsäure bis zum Sieden.

Tinctura Ferri chlorat. aeth.

im Wasserbade mit Salzsäure erwärmt, bis Aether und Alkohol verdampft sind, darauf mit Permanganatlösung versetzt etc.

a. $8,800 \mathrm{~g}=0,10136 \mathrm{~g}(18,1 \mathrm{ccm} \times 0,0056)=1,15$ Proc. Fe.

b. $10,805=0,13776 \mathrm{~g}(24,6 \mathrm{ccm} \times 0,0056)=1,27$ Proc. Fe.

Tinctura Ferri acet. aeth.

mit Salzsäure versetzt und so lange gekocht, bis der Essigäther und der Alkohol verdampft sind, darauf mit Wasser verdünnt etc.

a. $4,6260 \mathrm{~g}=0,18256 \mathrm{~g}(32,6 \mathrm{ccm} \times 0,0056)=3,94$ Proc. Fe.

b. $4,407.0 \mathrm{~g}=0,16464 \mathrm{~g}(29,4 \mathrm{ccm} \times 0,0056)=3,74$ Proc. Fe.

Tinctura Ferri chlorati.

In $100\left(\mathrm{FeCl}^{2}+20\right.$ Proc. $\left.\mathrm{H}^{2} \mathrm{O}\right)$ sind $35,55 \mathrm{Fe}$ enthalten, in 25 also $8,8875 \mathrm{Fe}$.

In 251 der Tinctur sind $25\left(\mathrm{FeCl}^{8}+20\right.$ Proc. $\left.\mathrm{H}^{2} \mathrm{O}\right)$, also 8,8875 oder 3,54 Proc. Fe.

a. $5,740 \mathrm{~g}=0,21056 \mathrm{~g}(37,6 \mathrm{ccm} \times 0,0056)=3,66$ Proc. Fe.

b. $4,1040 \mathrm{~g}=0,1456 \mathrm{~g}(26 \mathrm{ccm} \times 0,0056)=3,54$ Proc. Fe.

Ammonium chloratum ferratum.

a. $1,3240 \mathrm{~g}=0,03584 \mathrm{~g}(6,4 \mathrm{ccm} \times 0,0056)=2,71$ Proc. Fe.

b. $1,4680 \mathrm{~g}=0,04368 \mathrm{~g}(7,2 \mathrm{ccm} \times 0,0056)=2,98$ Proc. Fe. 
C. Schacht, Quantitative Bestimmung des Eisens auf jodometr. Wege. 909

Ferrum jodatum saccharatum.

a. $1,1420 \mathrm{~g}=0,03808 \mathrm{~g}(6,8 \mathrm{ccm} \times 0,0056)=18,49$ Proc. FeJ ${ }^{2}$.

b. $1,5820 \mathrm{~g}=0,05264 \mathrm{~g}(9,4 \mathrm{ccm} \times 0,0056)=18,38$ Proc. Fe $J^{2}$.

Ferrum sulfuricum.

Man nimmt nicht mehr als 0,5 g zur Eisenbestimmung. Auch thut man gut, stets dieselben Mengen verdünnter Schwefelsäure und Wasser zu nehmen. Das überschüssig zugesetzte Permanganat entfernt man durch Zusatz von etwas Oxalsäurelösung.

$0,4960 \mathrm{~g}$ gaben $0,09912 \mathrm{~g}(17,7 \mathrm{ccm} \times 0,0056)$

$278: 56=0,4960: 0,09991$

$0,09991: 0,09912=100: 99,2$ Proc. Fe.

Ferrum pulveratum.

a. $0,1070 \mathrm{~g}=0,10304 \mathrm{~g}(18,4 \mathrm{ccm} \times 0,0056)=96,3$ Proc. Fe.

b. $0,1070 \mathrm{~g}=0,1036 \mathrm{~g}(18,5 \mathrm{ccm} \times 0,0056)=96,8$ Proc. Fe.

c. $0,1005 \mathrm{~g}=0,09688 \mathrm{~g}(17,3 \mathrm{ccm} \times 0,0056)=96,4$ Proc. Fe.

Ferrum reductum.

Man thut gut, nicht mehr als $0,1 \mathrm{~g}$ des Präparates, $5-10 \mathrm{ccm}$ verdünnte Schwefelsäure, $1 \mathrm{~g}$ Jodkalium zu nehmen.

a. $0,1 \mathrm{~g}=0,09408 \mathrm{~g}=94,08$ Proc. Fe.

b. $0,1 \mathrm{~g}=0,09408 \mathrm{~g}=94,08$ Proc. Fe.

c. $0,1015 \mathrm{~g}=0,09405 \mathrm{~g}=94,05$ Proc. Fe.

d. $0,1135 \mathrm{~g}=0,09424 \mathrm{~g}=94,24$ Proc. Fe.

Von einem andern Präparate wurden $2,0210 \mathrm{~g}$ in Salzsäure gelöst und auf $500 \mathrm{ccm}$ aufgefüllt. $25 \mathrm{ccm}=0,10105$.

a. $17,55 \mathrm{ccm}=0,09828 \mathrm{~g}=97,26$ Proc. Fe.

b. $17,6 \mathrm{ccm}=0,09856 \mathrm{~g}=97,53$ Proc. Fe.

Ausserdem habe ich in folgenden Eisenpräparaten das Eisen auf jodometrischem Wege bestimmt, nachdem ich dasselbe durch Behandeln mit Salzsäure und Kaliumchlorat in Eisenchlorid übergeführt hatte.

Ferrum pyrophosphoricum cum Ammonio citrico.

a. $0,5670 \mathrm{~g}=0,08624 \mathrm{~g}(15,4 \mathrm{ccm} \times(0,0056)=15,21$ Proc. Fe.

b. $0,5120 \mathrm{~g}=0,07896 \mathrm{~g}(14,1 \mathrm{ccm} \times 0,0056)=15,42$ Proc. Fe.

Ferrum phosphoricum oxydulatum.

a. $0,5100 \mathrm{~g}=0,17304 \mathrm{~g}(30,9 \mathrm{ccm} \times 0,0056)=33,93$ Proc. Fe.

b. $0,5510 \mathrm{~g}=0,18984 \mathrm{~g}(33,9 \mathrm{ccm} \times 0,0056)=34,4$ Proc. Fe. 
910 C. Schacht, Quantitative Bestimmung des Eisens auf jodometr. Wege.

Ferrum citricum oxydatum.

a. $0,8500 \mathrm{~g}=0,17640 \mathrm{~g}(31,5 \mathrm{ccm} \times 0,0056)=20,75$ Proc. Fe.

b. $0,5735 \mathrm{~g}=0,12096 \mathrm{~g}(21,6 \mathrm{ccm} \times 0,0056)=21,06$ Proc. Fe.

Ferrum citricum ammoniatum.

a. $0,8580 \mathrm{~g}=0,13160 \mathrm{~g}(23,5 \mathrm{ccm} \times 0,0056)=15,33$ Proc. Fe.

b. $0,8490 \mathrm{~g}=0,13328 \mathrm{~g}(23,8 \mathrm{ccm} \times 0,0056)=15,68$ Proc. Fe.

Ferrum peptonatum.

Verascht und mit Salzsäure und Kaliumchlorat behandelt.

a. $0,6120 \mathrm{~g}=0,04032 \mathrm{~g}(7,2 \mathrm{ccm} \times 0,0056)=6,58$ Proc. Fe.

b. $0,6355 \mathrm{~g}=0,03976 \mathrm{~g}(7,1 \mathrm{ccm} \times 0,0056)=6,25$ Proc. Fe.

c. $0,6500 \mathrm{~g}=0,04144 \mathrm{~g}(7,4 \mathrm{ccm} \times 0,0056)=6,38$ Proc. Fe.

Ferrum albuminatum liquidum.

Verascht etc. wie bei Ferrum peptonatum.

a. 2,1620 $\mathrm{g}=0,00952 \mathrm{~g}(1,7 \mathrm{ccm} \times 0,0056)=0,44$ Proc. Fe.

b. $3,4410 \mathrm{~g}=0,01624 \mathrm{~g}(2,9 \mathrm{ccm} \times 0,0056)=0,47$ Proc. Fe.

c. $3,0830 \mathrm{~g}=0,0140 \mathrm{~g}(2,5 \mathrm{ccm} \times 0,0056)=0,45$ Proc. Fe.

Pilulae Ferri carbonici.

Verascht etc. wie bei Ferrum peptonatum.

a. $0,7270 \mathrm{~g}=0,05264 \mathrm{~g}(9,4 \mathrm{ccm} \times 0,0056)=7,24$ Proc. Fe.

b. $1,1605 \mathrm{~g}=0,08848 \mathrm{~g}(15,8 \mathrm{ccm} \times 0,0056)=7,54$ Proc. Fe.

Tinctura Ferri pomati, wie bei Ferrum peptonatum.

a. $7,2170 \mathrm{~g}=0,09408 \mathrm{~g}(16,8 \mathrm{ccm} \times 0,0056)=1,30$ Proc. Fe.

b. $8,3900 \mathrm{~g}=0,12656 \mathrm{~g}(22,6 \mathrm{ccm} \times 0,0056)=1,50$ Proc. Fe.

Syrupus Ferri jodati, wie bei Ferrum peptonatum.

a. 6,8930 $\mathrm{g}=0,04648 \mathrm{~g}(8,3 \mathrm{ccm} \times 0,0056)=0,68$ Proc. Fe.

$56: 320=0,68: 3,77$ Proc. Fe J'.

b. $6,6810 \mathrm{~g}=0,04704 \mathrm{~g}(8,4 \mathrm{ccm} \times 0,0056)=0,70$ Proc. $\mathrm{Fe}$ $=3,87$ Proc. $\mathrm{FeJ}^{2}$.

c. $5,9460 \mathrm{~g}=0,04312 \mathrm{~g}(7,7 \mathrm{ccm} \times 0,0056)=0,72$ Proc. $\mathrm{Fe}$ $=3,98$ Proc. FeJ ${ }^{2}$. 